\title{
Extracellular Tau Oligomers Induce Invasion of Endogenous Tau into the Somatodendritic Compartment and Axonal Transport Dysfunction
}

\author{
Eric Swanson ${ }^{\mathrm{a}}$, Leigham Breckenridge ${ }^{\mathrm{a}}$, Lloyd McMahon ${ }^{\mathrm{b}}$, Sreemoyee Som ${ }^{\mathrm{c}}$, Ian \\ McConnell ${ }^{\mathrm{a}}$, and George S. Bloom ${ }^{\mathrm{a}, \mathrm{b}, \mathrm{d},{ }^{*}}$ \\ aDepartment of Biology, University of Virginia, Charlottesville, VA, USA \\ bDepartment of Cell Biology, University of Virginia, Charlottesville, VA, USA \\ 'Department of Biomedical Engineering, University of Virginia, Charlottesville, VA, USA \\ ${ }^{\mathrm{d} D e p a r t m e n t}$ of Neuroscience, University of Virginia, Charlottesville, VA, USA
}

\begin{abstract}
Aggregates composed of the microtubule associated protein, tau, are a hallmark of Alzheimer's disease and non-Alzheimer's tauopathies. Extracellular tau can induce the accumulation and aggregation of intracellular tau, and tau pathology can be transmitted along neural networks over time. There are six splice variants of central nervous system tau, and various oligomeric and fibrillar forms are associated with neurodegeneration in vivo. The particular extracellular forms of tau capable of transferring tau pathology from neuron to neuron remain ill defined, however, as do the consequences of intracellular tau aggregation on neuronal physiology. The present study was undertaken to compare the effects of extracellular tau monomers, oligomers, and filaments comprising various tau isoforms on the behavior of cultured neurons. We found that $2 \mathrm{~N} 4 \mathrm{R}$ or 2N3R tau oligomers provoked aggregation of endogenous intracellular tau much more effectively than monomers or fibrils, or of oligomers made from other tau isoforms, and that a mixture of all six isoforms most potently provoked intracellular tau accumulation. These effects were associated with invasion of tau into the somatodendritic compartment. Finally, we observed that $2 \mathrm{~N} 4 \mathrm{R}$ oligomers perturbed fast axonal transport of membranous organelles along microtubules. Intracellular tau accumulation was often accompanied by increases in the run length, run time and instantaneous velocity of membranous cargo. This work indicates that extracellular tau oligomers can disrupt normal neuronal homeostasis by triggering axonal tau accumulation and loss of the polarized distribution of tau, and by impairing fast axonal transport.
\end{abstract}

\section{Keywords}

Alzheimer's disease; axonal transport; BACE1; brain derived neurotrophic factor; neuropeptide Y; tau; tauopathies

"Correspondence to: George S. Bloom, Department of Biology, University of Virginia, PO Box 400328, Charlottesville, VA, 22904-4328, USA. Tel.: +1 434243 3543; gsb4g@ virginia.edu.

SUPPLEMENTARY MATERIAL

The supplementary material is available in the electronic version of this article: http://dx.doi.org/10.3233/JAD-170168. 


\section{INTRODUCTION}

Hyperphosphorylated, poorly soluble aggregates of the neuron-specific, microtubule (MT)associated protein, tau, define a set of neurodegenerative disorders known as tauopathies, of which Alzheimer's disease (AD) is the most prevalent [1]. It has long been known that tau pathology exhibits a time-dependent spread along defined neuronal pathways as $\mathrm{AD}$ progresses, and that the extent of this progression correlates with cognitive decline [2]. Recent studies have demonstrated that extracellular tau can be internalized by cells and propagate along synaptically connected neuronal networks in vivo [3-5]. In vitro studies have also demonstrated that extracellular tau can induce the aggregation of intracellular tau, which can then be released from the cell and taken up by other cells [6-8]. Taken as a whole, these data have led to the hypothesis that the propagation of tau pathology is mediated by a prion-like process in which extracellular pathological tau enters a naive cell, whereupon it seeds pathogenic conformational changes in the pool of monomeric internal tau that can then be transmitted again to interconnected neurons [9-13]. While the evidence for this seededrecruitment phenomena has expanded greatly in recent years, selective vulnerability of certain neuronal populations has also been postulated as an important contributing factor for this well-defined disease progression [14].

Several important issues remain unresolved, however, including which forms of extracellular tau induce intracellular tau aggregation, and how that aggregation alters neuronal behavior. Because neurons do not divide and are more difficult to culture than constitutively dividing non-neuronal cell lines, and because wild type tau is not prone to aggregate on its own, most prior in vitro studies of the effects of extracellular tau on intracellular tau have utilized immortalized, non-neuronal cells over-expressing truncated or otherwise mutated forms of aggregation-prone tau $[6,7,15]$. Additionally, while it was initially thought that the poorly soluble filaments of hyperphosphorylated tau found in tauopathies represent the most toxic forms of tau, there is growing evidence that prefibrillar, soluble tau oligomers [16-18] mediate synaptotoxicity, cellular dysfunction, and neuron death in murine and cultured neuron models of AD [18-22]. Soluble tau oligomers may therefore resemble oligomers of the prion protein $(\mathrm{PrP})$, which have been shown to exhibit a greater capacity than fibrillar PrP species to seed misfolding of monomeric PrP [28].

In the central nervous system, tau is a predominantly axonal protein that is expressed as six isoforms resulting from the alternative splicing of a single tau gene (MAPT) per haploid chromosome set. This results in tau isoforms containing 0,1 , or $2 \mathrm{~N}$-terminal inserts, and 3 or $4 \mathrm{C}$-terminal MT binding repeats [23, 24]. We refer to these isoforms as $0 \mathrm{~N} 3 \mathrm{R}, 1 \mathrm{~N} 3 \mathrm{R}$, 2N3R, 0N4R, 1N4R, and 2N4R tau. Interestingly, the isoform composition of tau aggregates varies among different tauopathies, such as corticobasal degeneration (predominantly $4 R$ ) [31], Pick's disease (predominantly 3R) [32], and AD (approximately equal amounts of 3R and $4 \mathrm{R}$ [33]). Moreover, intronic mutations resulting in the alteration of tau isoform ratios, but not protein levels, can be fully penetrant for neurological disorders, indicating that isoforms play an important role in tau pathology [25]. 
Finally, while cell-to-cell transmission of pathological tau is well established, the functional consequences of intracellular tau aggregation to neuronal physiology have attracted little attention. Beyond its role in stabilizing MTs, tau has also been shown to play an important role in the regulation of fast axonal transport. MT-bound tau is known to act as a "speed bump" for kinesin-based transport, and there is an increased propensity for MT motors to detach or pause in the case of kinesin, or reverse direction in the case of cytoplasmic dynein, upon contact with tau-enriched regions of MTs [26-28]. It has further been demonstrated that kinesin motors in vitro make longer runs and have a higher motive force in the absence of tau [29], but little is known about how a disruption or loss of tau may alter this MT motor transport inside living neurons.

With this background in mind, the current study was undertaken to test whether extracellular tau oligomers affect the endogenous intracellular tau distribution and the behavior of cultured mouse cortical neurons. We demonstrate that the ability of extracellular tau oligomers to drive accumulation of intracellular tau varies by the isoform composition of the oligomers, that oligomers can cause dramatic invasion of endogenous intracellular tau into the somatodendritic compartment, and finally, that these effects are associated with the dysregulation of MT-based fast axonal transport. Altogether, our results imply that extracellular tau oligomers induce a breakdown of normal neuronal homeostasis that represents an early stage in neurodegeneration.

\section{MATERIALS AND METHODS}

\section{Recombinant tau}

Expression vectors for all six wild-type human tau isoforms with a $5^{\prime}$-His-tag (kindly provided by the late Lester "Skip" Binder) were transformed into BL21 cells and purified by affinity chromatography utilizing a HiTrap $\mathrm{Ni}^{2+}$ column (GE Healthcare) and imidazole gradient according to the manufacturer's instructions. Recombinant protein was then concentrated in $10 \mathrm{~K}$ molecular weight cut off spin columns (Millipore) and dialyzed into phosphate buffer, $\mathrm{pH}$ 7.4.

\section{Oligomer production}

Oligomeric tau was prepared as described previously by another group [17]. Tau was brought to $4 \mu \mathrm{M}$ in $100 \mathrm{mM}$ Tris, $0.1 \mathrm{mM}$ EDTA, and $150 \mu \mathrm{M}$ Tris(2-carboxy ethyl)phosphine (TCEP; Life Technologies) and treated overnight with $50 \mu \mathrm{M}$ benzophenone-4-maleimide (B4M; Sigma-Aldrich). The protein was then treated with $5 \mathrm{mM}$ dithiothreitol (DTT; Roche) to inactivate B4M and dialyzed overnight into $100 \mathrm{mM}$ Tris, 0.1 $\mathrm{mM}$ EDTA, and $5 \mathrm{mM}$ DTT. A portion was removed for monomer treatments, and the protein was then allowed to aggregate overnight in the presence of $150 \mu \mathrm{M}$ arachidonic acid (AA; Invitrogen), after which the protein was treated for 5 min with UV light at $254 \mathrm{~nm}$ (Spectroline model EF-180). Oligomers were used within 2 weeks of preparation. B4M- and AA-treated protein not exposed to UV was used as the fibrillar tau preparation. 


\section{Antibodies}

The following antibodies were used: Alz50 and MC1 (conformation-sensitive mouse monoclonals to tau; provided by Dr. Peter Davies of the Feinstein Institute for Medical Research); chicken anti-MAP2 (Abcam, catalog \# ab5392); PHF1 (mouse monoclonal to tau phosphorylated at S396/S404; provided by Dr. Peter Davies; Tau 5 (mouse monoclonal pantau; provided by the late Dr. Lester "Skip" Binder of Michigan State University); T14 (mouse monoclonal to human tau; provided by Drs. Virginia Lee and John Trojanowski of

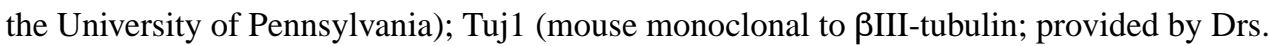
Tony Spano and Tony Frankfurter of the University of Virginia); AlexaFluor 488 goat antimouse IgG (Invitrogen catalog \# A-11001); AlexaFluor 594 goat anti-mouse IgG (Invitrogen catalog \# A-11005); AlexaFluor 488 goat anti-chicken IgG (Invitrogen catalog \# A-11039).

\section{Western blots}

$2 \mu \mathrm{g}$ total protein were separated on 4-12\% gradient Bis-Tris SDSPAGE gels (Invitrogen) for $2 \mathrm{~h}$ and transferred to a nitrocellulose membrane. Membranes were blocked in Licor TBS blocking buffer (Licor) and treated with primary antibodies overnight. Membranes were then incubated with infrared-tagged secondary antibodies (Licor), and analyzed using a Licor Odyssey imaging system.

\section{Electron microscopy}

Samples of monomeric, oligomeric, and fibrillar tau at $4 \mu \mathrm{M}$ total tau each were adsorbed overnight to 300 mesh copper EM grids (Electron Microscopy Services), counter-stained with uranyl acetate and imaged on a JEOL 1010 transmission electron microscope equipped with a 16 megapixel cooled CCD (model SAI-12c, Scientific Instruments and Applications, Inc.).

\section{Primary neurons}

Cortical neurons were isolated from wild type (C57/B16) and tau-knockout [30] embryos aged 16.5-18.5 days as previously described [31]. Briefly, brains were removed from the embryos and placed in Hank's balanced salt solution (HBSS; Invitrogen) in a sterile environment, after which the cortical tissue was isolated following removal of the meninges. The tissue was then digested at $37^{\circ} \mathrm{C}$ in $0.25 \%$ Trypsin for $45 \mathrm{~min}$. The trypsin protease activity was quenched with heat-inactivated fetal bovine serum (FBS; HyClone/GE Healthcare), and the cells were washed 3 times with warm HBSS and treated with 100 units DNAse II (Worthington) per brain. The cells were then mechanically dissociated with Pasteur pipettes, diluted in Neurobasal media containing serum-free neuron supplement B27, 1-glutamine, glucose, penicillin/streptomycin (all from Invitrogen) and 5\% FBS, and plated at roughly $6250 \mathrm{cells} / \mathrm{cm}^{2}$. Plating media was removed $3 \mathrm{~h}$ later and replaced with plating media lacking FBS.

\section{Extracellular tau treatment}

Primary cortical neurons were treated at 10 days in vitro with the indicated monomeric, oligomeric or fibrillar tau species at a final concentration of $50 \mathrm{nM}$ total tau for $18 \mathrm{~h}$. Cells were washed with PBS prior to immunofluorescence staining. 


\section{Immunofluorescence labeling}

Primary neuron cultures were fixed at room temperature (RT) for $15 \mathrm{~min}$ in $3.7 \%$ paraformaldehyde, washed 3 times in cold PBS, and permeabillized in $0.2 \%$ TritonX-100 (Sigma) in PBS for 10 min. Cells were again washed in PBS, followed by blocking in 2\% bovine serum albumin (Sigma)/0.1\% Tween-20 (Fisher) in PBS for 30 min and incubation with the primary antibodies overnight. Cells were then treated with secondary antibodies labeled with Alexafluor-488 or Alexafluor-568; (Life Technologies) for $1 \mathrm{~h}$ at RT. After a final series of brief washes in PBS, the coverslips were mounted on glass slides using Fluormount G (Fisher).

CVN mice, a model for AD [32], were deeply anesthetized and perfused transcardially with PBS for 5 min followed by $4 \%$ paraformaldehyde for $5 \mathrm{~min}$, after which the brains were removed. Tissue was dehydrated, embedded in paraffin, and cut into $5 \mu \mathrm{m}$ thick sections that were adsorbed onto glass slides. The sections were deparaffinized using xylenes, rehydrated in a graded series of ethanol:water washes from $100 \%$ ethanol to $100 \%$ water, blocked for 30 $\mathrm{min}$ in 5\% FBS/0.2\% Tween 20 and incubated overnight with primary antibody (Alz50 or $\mathrm{MC1}$ ). Following 3 PBS washes to remove excess primary antibody, the sections were then incubated with secondary antibodies labeled with Alexafluor-488 goat anti-mouse IgG for 1 $\mathrm{h}$ at RT. Finally, the tissue sections were washed twice with PBS, incubated for $5 \mathrm{~min}$ with 200 nM DAPI in PBS to stain nuclei, washed once more with PBS, and then overlaid with glass coverslips that were secured to the glass slides with Fluormount G.

\section{Quantitative light microscopy of tau aggregation}

Neurons labeled with Tau-5, a monoclonal antibody that recognizes all tau isoforms independently of post-translational modifications [33], were imaged using a 20X 0.4 NA objective on an EVOS FL cell imaging system (ThermoFisher Scientific) at a constant level of illumination. Immunofluorescence micrographs were analyzed using FIJI software (also known as ImageJ2: http://imagej.net/ImageJ2) with an algorithm adapted from a previously described protocol [42]. Images were thresholded at the mean setting ([maximum fluorescence-minimum fluorescence]/2) and pixel groups were then segregated into contiguous groups larger or smaller than 10,000 utilizing the "analyze particles" function in FIJI [43]. The area of groups smaller than 10,000 contiguous pixels divided by the total area above the threshold intensity $\mathrm{x} 100$ gives the value for percent accumulation/disruption of the protein of interest. Groups smaller that 10,000 are pseudo-colored red and groups larger than 10, 000 are pseudo-colored green to assist with visualization.

\section{Fast axonal trafficking analysis}

Neurons were transfected with fluorescent expression vectors for BDNF-mRFP1, NeuropeptideY-mCherry (both kindly provided by Dr. Michael Silverman of Simon Fraser University) or BACE1-mRFP1 (kindly provided by Dr. Huaye Zhang of Rutgers Robert Wood Johnson Medical School) utilizing Lipofectamine 2000 transfection reagent (Invitrogen). Cells were transfected 3 days prior to imaging, then vesicle movement was visualized upon excitation at $568 \mathrm{~nm}$ with a Nikon 60X planapo objective mounted on Nikon Eclipse Ti inverted microscope equipped with a Yokogawa spinning disc confocal head, and a Plexiglas chamber that maintained an atmosphere of $37^{\circ} \mathrm{C}$ and $5 \% \mathrm{CO}_{2}$. Images were 
taken at 2 frames per second. Axons were identified as neurites of uniform length with minimal to no branching, and directionality was determined by tracing them to a synapse or neuronal perikaryon of origin. The movement of fluorescent vesicles was analyzed as kymographs utilizing the ImageJ plug-in "Multiple Kymograph" (European Molecular Biology, Heidelberg, Germany). Run length and run time were derived from the kymographs, as was "instantaneous velocity", which we define as the average velocity of the vesicle while in apparently continuous motion. Velocity was taken for all slopes in the kymograph, while run time and run length were reported only for organelles whose motility began and ended during the imaging timeframe.

\section{Statistical analysis}

Data were analyzed using GraphPad Prism software by one-way analysis of variance, with a Bonferonni post-test to analyze variance between treatments.

\section{RESULTS}

\section{Production of tau oligomers}

Formation of oligomers from recombinant versions of each of the six human CNS tau isoforms was confirmed by western blotting (Fig. 1A-C). Tau oligomerized by this protocol (see Materials and Methods) has previously been shown to migrate on western blots similarly to soluble multiple- $n$ tau species isolated from AD brain [17]. Negative stain, transmission electron micrographs of monomeric, oligomeric, and fibrillar 2N4R tau used throughout this study are shown in Fig. 1D.

\section{Extracellular tau oligomers induce endogenous tau redistribution and accumulation}

Previous studies have established that extracellular tau filaments can cause intracellular tau fragments corresponding to the MT-binding repeat domains to accumulate as puncta in nonneuronal cells stably transfected to express the tau fragments [6, 7]. Extracellular tau filaments have also been demonstrated to cause tau aggregation in neurons expressing mutant tau $[8,34]$. To determine if extracellular tau oligomers can similarly drive accumulation of endogenous full length tau in cultured mouse neurons, we utilized a quantitative assay for accumulation of axonal tau (see Materials and Methods). Immunofluorescence micrographs were first thresholded by intensity to eliminate background fluorescence, after which each set of contiguous pixels above the threshold value was assigned to a group that was either larger or smaller than a pre-defined number of contiguous pixels. As the immunofluorescence staining becomes discontinuous there is an increase in smaller pixel groups, indicative of tau accumulation (Fig. 2A). After initially testing cutoffs between 1,000 and 20,000 contiguous pixels (Fig. 2B), we chose 10,000 pixels of contiguous labeling as the cutoff between continuous and discontinuous tau staining, which are pseudo-labeled green and red, respectively, throughout this report. Antibodies specific for human tau did not stain the cells above background levels (Fig. 2C), indicating that the accumulated and missorted intracellular tau was pre-dominantly or exclusively endogenous mouse tau. As can be seen in Fig. 3, extracellular tau oligomers also caused extensive accumulation of intracellular tau and its conspicuous invasion into the somatodendritic compartment. 
Using this quantification method, we found that untreated neurons, and neurons treated for $18 \mathrm{~h}$ with monomeric or fibrillar $2 \mathrm{~N} 4 \mathrm{R}$ tau at $50 \mathrm{nM}$ total tau had $20 \%, 19 \%$, and $17 \%$ endogenous tau accumulation, respectively. In contrast, cells treated comparably with oligomeric tau showed an average of $34 \%$ tau accumulation, an $~ 1.75$-fold increase over the other conditions tested (Fig. 4A, B, Supplementary Table 1).

To determine whether intracellular tau accumulation represented a global disruption of MTs, neurons exposed to extracellular 2N4R tau under the same conditions were labeled with antibodies to MAP2, a somatodendritic protein $[46,47]$ that shares a highly conserved MTbinding repeat region with tau [35], and to $\beta$ III-tubulin, a neuron-specific tubulin isoform [36]. Neither MAP2 (Fig. 4C, D) nor $\beta$ III-tubulin (Fig. 4E, F) showed signs of accumulation in response to extracellular monomeric, oligomeric or fibrillar tau. These data imply that extracellular tau oligomers alter the distribution of endogenous tau without affecting MTs in any other ways that might have been detected by our methods.

\section{Extracellular tau oligomers do not rapidly induce AD-like conformational change or phosphorylation of endogenous intracellular tau}

Cells treated with $2 \mathrm{~N} 4 \mathrm{R}$ tau oligomers were also analyzed by immunofluorescence using a panel of anti-tau antibodies that detect conformational or phosphorylation-dependent changes characteristic of pathological tau in $\mathrm{AD}$ neurons. Cells were stained with the conformation-specific antibodies, Alz50 [50, 51] and MC1 [52], which detect early pathology-associated changes in tau misfolding, and PHF1, which recognizes tau that is phosphorylated at S396/S404 [53] and is enriched in late stage tau inclusions [37]. No immunoreactivity was observed with Alz50 or MC1 in untreated neurons or in neurons treated with tau monomers or oligomers (Fig. 5), although as shown in Supplementary Figure 1, both antibodies labeled misfolded tau in brain sections obtained from the CVN strain of AD model mice [32]. In contrast to what was observed for Alz50 and MC1 a low, constant level of PHF1 immunoreactivity was observed in cultured neurons regardless of whether or not they were exposed to extracellular tau monomers or oligomers (Fig. 5).

\section{Isoform composition of tau oligomers affects the extent of intracellular tau accumulation}

To determine whether accumulation of endogenous tau depends on the isoform composition of the externally applied tau oligomers, we repeated these experiments with the remaining five isoforms of CNS tau. Oligomeric 2N3R tau was able to induce statistically significant accumulation of endogenous tau, albeit not quite as effectively as 2N4R tau (Fig. 6A). Oligomeric $0 \mathrm{~N} 4 \mathrm{R}$ tau induced accumulation of endogenous tau to a small, though significant extent, while oligomeric $0 \mathrm{~N} 3 \mathrm{R}, 1 \mathrm{~N} 3 \mathrm{R}$, and $1 \mathrm{~N} 4 \mathrm{R}$ tau did not significantly stimulate accumulation of intracellular tau (Fig. 6B, C).

Finally, neurons were exposed to extracellular tau of two mixed isoform compositions, a 1:1 mixture of 2N3R and 2N4R tau, and all six isoforms in equimolar ratios. Total tau concentrations for each experiment were held constant at $50 \mathrm{nM}$ and isoform mixtures were allowed to oligomerize together. The 1:1 mixture of 2N3R:2N4R did not alter the tau distribution when presented to neurons as monomers or fibrils. In contrast, when presented as oligomers, the 1:1 mixture did cause a significant increase in tau accumulation, although 
to a lesser extent than observed for either 2N3R or 2N4R oligomers alone (Fig. 7A, Supplementary Table 1). Interestingly, the most potent response was seen upon treatment with all six isoforms, as both monomeric and fibrillar treatments caused an increase in tau mislocalization relative to no tau treatment, and oligomeric tau had an even larger, statistically significant effect (Fig. 7B, Supplementary Table 1). It is also noteworthy that oligomers made from all six isoforms caused disruption of MAP2 as well (Fig. 7B), indicating that this treatment represents a particularly toxic insult to neurons.

\section{Accumulation of endogenous axonal tau is associated with dysregulation of fast axonal transport}

In prior studies of cell-to-cell propagation of tau, little analysis has been performed regarding effects of tau accumulation on neuronal physiology. As a predominantly axonal protein, tau is known to regulate the attachment and movement of various motors involved in axonal transport, and this effect has been shown to affect kinesin-1 more potently than cytoplasmic dynein [28]. More specifically, tau appears to act as an obstacle, or "speed bump", that can reduce cargo run lengths [26-28] and increase the likelihood of the motor disengaging from MTs $[28,29]$. To analyze the effect, if any, that the mislocalization of tau we describe here has on axonal transport, primary neurons were transfected with fluorescently-tagged proteins to label vesicles undergoing MT-directed fast axonal transport. Specifically, BDNF-mRFP1 and BACE1-mRFP1 were utilized to analyze effects on kinesin-1-dependent transport [55, 56], and NeuropeptideY-mCherry to observe kinesin-3dependent transport [55] (see Supplementary Movie 1). Because 2N4R tau oligomers produced a robust response in previous experiments and data analysis for these experiments was extremely time consuming, we focused only on 2N4R tau oligomers. Trafficking of the various fluorescently labeled vesicles was analyzed by live cell, time lapse imaging. 1-2\% of neurons expressed the fluorescent fusion proteins, allowing for the imaging of isolated neurons. Axons were identified by finding regions of consistent thickness and low branching, which were then traced back to their cell body of origin to discriminate anterograde versus retrograde transport. Vesicle transport was analyzed by kymograph analysis, with values obtained for run length, run time and velocity while in motion (Fig. 8 and Supplementary Table 2).

BDNF-mRFP1 labeled vesicles showed no significant changes in run length, run time, or instantaneous velocity in either the anterograde or retrograde direction upon treatment with monomeric full-length tau, as compared to a non-treated control. Neurons treated with oligomeric tau showed an $\sim 2.5$-fold increase in anterograde and retrograde run length, an $\sim 30 \%$ increase in anterograde run time and an $\sim 2$-fold increase in retrograde time, and an $\sim 1.5$-fold increase in anterograde instantaneous velocity. Similar results were obtained for neurons transfected with BACE1-mRFP1, a protease that helps produce amyloid- $\beta$ (A $\beta$ ) from the amyloid- $\beta$ protein precursor (A $\beta P P$ ), and has been shown to be trafficked along with A $\beta$ PP by kinesin-1 [56]. BACE1-mRFP1 expressing neurons treated with oligomeric tau showed an $\sim 2$-fold increase in anterograde run length and an $\sim 40 \%$ increase in anterograde instantaneous velocity, suggesting that oligomeric $2 \mathrm{~N} 4 \mathrm{R}$ tau treatments affect multiple kinesin-1 cargoes in the same general manner. 
Otherwise identical experiments were also performed using mCherry-tagged NeuropeptideY, which is trafficked anterogradely by a different motor, kinesin-3 [55]. No significant alterations were seen for anterograde run length, run time, or velocity when comparing untreated, 2N4R monomer-treated, and 2N4R oligomer-treated cells. 2N4R oligomers did not affect retrograde run time either, but they did increase retrograde run length and velocity by $\sim 2.3$-fold and $\sim 20 \%$, respectively (Fig. $8 \mathrm{D}$ ). Taken together, these results indicate that tau mislocalization following oligomeric tau treatment of neurons leads to dysregulation of axonal transport for multiple types of cargoes.

To determine whether these effects of extracellular tau oligomers on axonal transport depend on intracellular tau, transport experiments were repeated using neurons isolated from tau knockout mice [30]. While the values for run time, run length and instantaneous velocity in untreated tau knockout neurons were slightly elevated relative to the control values in wild type neurons, with minor exceptions, no changes were seen in these parameters for any of the three cargos in the anterograde or retrograde direction after cellular exposure to $2 \mathrm{~N} 4 \mathrm{R}$ tau monomers or oligomers (Fig. 9). The only exceptions were a small, but significant changes in the retrograde run length and run time for BDNF-transfected cells and retrograde velocity for NeuropeptideY transfected cells when treated with monomeric tau (Fig 9C, Supplementary Table 2). The collective results of the organelle transport experiments in wild type and tau knockout neurons support the hypothesis that extracellular tau oligomers disrupt anterograde and retrograde fast axonal transport by causing accumulation of endogenous intracellular tau.

\section{DISCUSSION}

A growing body of evidence from human $\mathrm{AD}$ brain [2], AD model mouse brain [3-5], and cultured cells $[6-8,34,38-40]$ has indicated that intracellular tau aggregation can be triggered by a prion-like mechanism $[10,12,41]$ following uptake of aggregated extracellular tau. Mechanistic insight into this process has come primarily from cultured cell studies, many of which have made use of extracellular filaments made from tau fragments corresponding to the MT-binding repeat domains (tau-RD) and non-neuronal cells stably transfected to express tau-RD. These collective studies have yielded impressive gains in understanding about the tau aggregation process, yet several other important issues have been largely ignored until now. Notable examples include the effects of aggregated extracellular tau on the endogenous full length tau in cultured neurons; the relative abilities of extracellular, full length oligomeric versus fibrillar tau to provoke intracellular tau accumulation; isoform-specific effects of the extracellular tau on intracellular tau aggregation; and perhaps most importantly, other cell biological responses of bona fide neurons to aggregation of their endogenous tau.

Here we document our efforts to shed light on all of these issues. Adapting a quantitative image analysis paradigm, we show that intracellular accumulation of the endogenous neuronal tau into apparent aggregates is induced more potently by extracellular oligomers than by extracellular filaments made from full length tau, that tau isoforms containing two $\mathrm{N}$-terminal inserts are more potent than isoforms containing 0 or $1 \mathrm{~N}$-terminal insert, and that mixtures of all six CNS tau isoforms are even more potent (Figs. 2, 4, 6, and 7). 
Importantly, the accumulation of tau does not seem to signify fragmentation of MTs, because with one exception, it is not accompanied by altered distribution of $\beta$ III-tubulin, a neuron-specific tubulin isoform [36] or of MAP2, a dendrite-specific MT-associated protein that comprises a MT-binding repeat region with high sequence identity to the comparable region of tau [35]. The lone exception concerns extracellular oligomers assembled from a mixture of all six CNS tau isoforms, which cause apparent disruption of both tau and MAP2 (Fig. 7B), and thus may be cytotoxic. We also demonstrate that extracellular tau oligomers can induce invasion of tau from its normal region of highest concentration, the axon, into the somatodendritic compartment, and that intracellular tau accumulation alters MT-based fast axonal transport. Altogether, these results imply that extracellular tau oligomers can disrupt normal neuronal homeostasis by mechanisms that depend on intracellular tau.

The present study extends an earlier report that extracellular tau oligomers can provoke intracellular tau aggregation [40] by demonstrating that oligomeric tau is much more potent in this regard than tau filaments. It is notable, however, that short extracellular tau filaments have been shown to be taken up by cultured neurons more efficiently than long tau filaments or monomeric tau [39]. These earlier reports, combined with the data presented here, imply that the prion-like propagation of tau pathology in vivo relies on small, readily diffusible forms of aggregated tau, such as oligomers and short filaments.

Two curious new findings reported here concern the isoform composition of the extracellular tau oligomers. First, by testing each of the six isoforms individually, we found that intracellular tau aggregation is promoted robustly by $2 \mathrm{~N} 3 \mathrm{R}$ or $2 \mathrm{~N} 4 \mathrm{R}$ tau oligomers, but is induced barely, if at all, by $0 \mathrm{~N}$ or $1 \mathrm{~N}$ tau isoforms (Figs. $4 \mathrm{~A}-\mathrm{C}$ and 6). The $2 \mathrm{~N}$-terminal inserts are encoded by MAPT exons 2 and 3, neither of which is expressed in $0 \mathrm{~N}$ tau and only the first of which is expressed in $1 \mathrm{~N}$ tau. Co-expression of exons 2 and 3 therefore seems to enhance the ability of extracellular tau oligomers to provoke intracellular tau accumulation. While we cannot exclude the possibility that exon 3 expression alone is required for this feature of tau, no such tau isoforms are known to occur naturally. Additionally, $0 \mathrm{~N}$ and $2 \mathrm{~N}$ isoforms comprising four $\mathrm{C}$-terminal MT binding repeats encoded by exon 10 demonstrated an increase in tau disruption when compared to like isoforms with only three MT binding repeats, indicating that $4 \mathrm{R}$ isoforms provoke a more potent response than $3 \mathrm{R}$ isoforms. Secondly, oligomers assembled from an equimolar cocktail of all six isoforms were found to be the most potent inducers of intracellular tau aggregation (Fig. 7B). Although we cannot offer any obvious explanations for these results, they might relate to any of several factors. These include, for example, the isoform composition of the endogenous intracellular tau in the neurons we studied, which is an approximately 1:1 ratio of 0N3R:0N4R tau (data not shown); the possible presence of extracellular oligomers that individually comprise more than one tau isoform; and potential synergistic effects of multiple oligomeric species, such as those that might form from a mixture of all six tau isoforms. In this context, it is noteworthy that intronic tau gene mutations that alter the ratio of 3R:4R tau without altering tau sequence or steady state tau levels can be fully penetrant for non-Alzheimer's tauopathies [25]. The pathogenic potential of wild type tau can thus be dramatically affected by isoform composition, which the data presented here further indicate, but the underlying mechanisms remain mysterious. 
$\mathrm{AD}$ tau has long been known to be phosphorylated at multiple sites that are rarely phosphorylated in normal tau [42-45], and to adopt immunologically sensitive conformational changes [46-49]. Interestingly, we found that extracellular tau oligomers did not alter the immunoreactivity of intracellular tau with two monoclonal antibodies, Alz50 $[47,48]$ and MC1 [46], that detect AD tau conformations, nor with the PHF1 monoclonal, which recognizes tau phosphorylated at S396/S404 [37, 50] (Fig. 5). While our study is isolated to effects in the first $24 \mathrm{~h}$ of tau oligomer exposure, it is possible that longer exposure to tau oligomers or other types of tau aggregates would induce pathological epitopes consistent with those seen in AD and non-Alzheimer's tauopathies in vivo.

One of the most striking effects of extracellular tau oligomers that we found is invasion of endogenous intraneuronal tau into the somatodendritic compartment (Fig. 3). This phenomenon mimics an early event in AD pathogenesis [51] and signifies breakdown of a key feature of neuronal polarity. Excess dendritic tau has been implicated in synaptotoxicity mediated by NMDA receptors [52], and is potentiated by tau acetylation [53] Movement of tau into dendrites has also been reported by others in a small fraction of cultured neurons exposed to $A \beta$ oligomers (A $\beta O s$ ) [54]. We have observed this phenomenon in some of our prior studies of $\mathrm{A} \beta \mathrm{O}$ s $[43,44]$ but have not commented on it until now. The contrast between $\mathrm{A} \beta \mathrm{O}$ s and tau oligomers for this activity is striking, though, because the vast majority of neurons exposed to tau oligomers in the current study accumulated somatodendritic tau. When considered together, the quantitatively different effects of $\mathrm{A} \beta \mathrm{Os}$ and tau oligomers on tau localization in the somatodendritic compartment raise the possibility that $\mathrm{A} \beta \mathrm{O}$ s drive formation of intracellular tau oligomers that can escape the neurons and then "infect" other neurons, whose endogenous tau then enters the somatodendritic compartment. This $\mathrm{A} \beta \mathrm{O}$-induced process might lead to a slow buildup of extracellular tau oligomers and correspondingly slow appearance of mislocalized endogenous tau. We envision that directly supplying extracellular tau oligomers to neurons, as was done in the current study, would dramatically increase the rate of endogenous tau mislocalization into the soma and dendrites.

Besides inducing intracellular tau missorting, extracellular tau oligomers altered several parameters of MT-based fast axonal transport in a cargo-specific manner. We measured run lengths, run times and instantaneous velocities for fluorescently tagged BDNF, BACE1 and neuropeptide $\mathrm{Y}$ in the anterograde and retrograde directions in both wild type and tau knockout neurons (Figs. 8 and 9, Supplementary Table 2, and Supplementary Movie 1). Because axonal MTs are almost uniformly polarized with their plus ends facing the axon terminal [55, 56], plus end-directed MT motors power anterograde transport, whereas minus end-directed motors are responsible for retrograde transport. More specifically, BDNF and BACE1 rely mainly on kinesin-1 as an anterograde motor [57, 58], neuropeptide $\mathrm{Y}$ uses kinesin-3 for anterograde transport [57] and all three cargoes presumably depend on cytoplasmic dynein for retrograde transport.

Both BDNF and BACE1 exhibited large increases in run length and instantaneous velocity for anterograde transport after exposure of wild type neurons to oligomers, but not monomers of extracellular tau. Retrograde run times for BDNF were also dramatically 
increased by extracellular tau oligomers. In contrast, tau oligomers caused increases in retrograde run length and instantaneous velocity for neuropeptide Y.

These results must be interpreted in light of prior reports of tau acting as a virtual "speed bump" that can dislodge motile cargo from MTs [26, 28, 29, 59]. Our data suggest that aggregation of intracellular tau causes the tau to dissociate from MTs, thereby removing the speed bumps, and permitting more rapid and longer range motile events in a manner that is sensitive to the combination of MT motors associated with the cargo. Interestingly, extracellular tau oligomers also caused small, but significant increases in retrograde run times for BDNF and instantaneous retrograde velocity for neuropeptide $\mathrm{Y}$ in tau knockout neurons. These effects in the absence of intracellular tau suggest that extracellular tau oligomers have signaling functions that are distinct from any effects they have on reorganizing intracellular tau.

It is worth considering what the long-term consequences of a boost in fast axonal transport may have on neurons. Kinesin-1 transports many cargo types in addition to BDNF and BACE1 [57], and at least some of those cargoes may need to be delivered to sites along the length of the axonal plasma membrane. The speed bump function of tau may enable such scattered cargo delivery, and removal of the speed bumps may bias delivery to toward the axon terminal. It is easy to imagine that such impaired axonal transport could gradually compromise neuron health as $\mathrm{AD}$ pathogenesis proceeds at the level of individual neurons.

The collective results presented here implicate tau oligomers in the spread of tau pathology from the extracellular space to neuronal cytoplasm, pointing to a likely role for these toxic species in the spread of AD and other tauopathies. By utilizing primary cultured neurons, we were able to tie extracellular tau oligomers to two early steps in neurodegeneration: mislocalization of endogenous tau into the soma and dendrites, and dysregulation of fast axonal transport. Soluble tau oligomers thus represent potential targets for early diagnosis and therapeutic intervention for $\mathrm{AD}$ and non-Alzheimer's tauopathies.

\section{Supplementary Material}

Refer to Web version on PubMed Central for supplementary material.

\section{Acknowledgments}

The authors would like to thank Dr. Michael Silverman for the BDNF-mRFP1 and Neuropeptide Y-mRFP1 expression vectors; Dr. Huaye Zhang for the BACE1-mCherry expression vector; Drs. Peter Davies, Virginia Lee, John Trojanowski, Tony Spano, Tony Frankfurter, and the late Lester "Skip" Binder for monoclonal antibodies; Horst Wallrabe, Erin Kodis, Lauren Saunders Rudenko, Shahzad Khan, and Drs. Dora Bigler-Wang, Andrés Norambuena, Antonia Silva, Chris Deppmann, John Lazo, and Beth Sharlow for their intellectual input throughout the course of this study; and Webb and Tate Wilson. This work was supported primarily by the Owens Family Foundation (GSB) and NIH/NIGMS training grant T32 GM008136, which provided 2 years of support for Eric Swanson. Minor support was also provided by Alzheimer's Association Zenith Fellowship ZEN-16-363266 (GSB), the Cure Alzheimer's Fund (GSB and John Lazo) and NIH/NIA grant RF1 AG051085 (GSB).

Authors' disclosures available online (http://j-alz.com/manuscript-disclosures/17-0168r1). 


\section{References}

1. Ballatore C, Lee VMY, Trojanowski JQ. Tau-mediated neurodegeneration in Alzheimer's disease and related disorders. Nat Rev Neurosci. 2007; 8:663-672. [PubMed: 17684513]

2. Braak H, Braak E. Neuropathological stageing of Alzheimer-related changes. Acta Neuropathol. 1991; 82:239-259. [PubMed: 1759558]

3. Clavaguera F, Bolmont T, Crowther RA, Abramowski D, Frank S, Probst A, Fraser G, Stalder AK, Beibel M, Staufenbiel M, Jucker M, Goedert M, Tolnay M. Transmission and spreading of tauopathy in transgenic mouse brain. Nat Cell Biol. 2009; 11:909-913. [PubMed: 19503072]

4. de Calignon A, Polydoro M, Suárez-Calvet M, William C, Adamowicz DH, Kopeikina KJ, Pitstick R, Sahara N, Ashe KH, Carlson GA, Spires-Jones TL, Hyman BT. Propagation of tau pathology in a model of early Alzheimer's disease. Neuron. 2012; 73:685-697. [PubMed: 22365544]

5. Liu L, Drouet V, Wu JW, Witter MP, Small SA, Clelland C, Duff K. Trans-synaptic spread of tau pathology in vivo. PLoS One. 2012; 7:e31302. [PubMed: 22312444]

6. Frost B, Jacks RL, Diamond MI. Propagation of tau misfolding from the outside to the inside of a cell. J Biol Chem. 2009; 284:12845-12852. [PubMed: 19282288]

7. Sanders DW, Kaufman SK, DeVos SL, Sharma AM, Mirbaha H, Li A, Barker SJ, Foley AC, Thorpe JR, Serpell LC, Miller TM, Grinberg LT, Seeley WW, Diamond MI. Distinct tau prion strains propagate in cells and mice and define different tauopathies. Neuron. 2014; 82:1271-1288. [PubMed: 24857020]

8. Guo JL, Lee VMY. Neurofibrillary tangle-like tau pathology induced by synthetic tau fibrils in primary neurons over-expressing mutant tau. FEBS Lett. 2013; 587:717-723. [PubMed: 23395797]

9. Bloom GS. Amyloid- $\beta$ and tau: The trigger and bullet in Alzheimer disease pathogenesis. JAMA Neurol. 2014; 71:505-508. [PubMed: 24493463]

10. Frost B, Diamond MI. Prion-like mechanisms in neurodegenerative diseases. Nat Rev Neurosci. 2009; 11:155-159. [PubMed: 20029438]

11. Holmes BB, Diamond MI. Prion-like properties of Tau protein: The importance of extracellular Tau as a therapeutic target. J Biol Chem. 2014; 289:19855-19861. [PubMed: 24860099]

12. Guo JL, Lee VMY. Cell-to-cell transmission of pathogenic proteins in neurodegenerative diseases. Nat Med. 2014; 20:130-138. [PubMed: 24504409]

13. Goedert M, Clavaguera F, Tolnay M. The propagation of prion-like protein inclusions in neurodegenerative diseases. Trends Neurosci. 2010; 33:317-325. [PubMed: 20493564]

14. Walsh DM, Selkoe DJ. A critical appraisal of the pathogenic protein spread hypothesis of neurodegeneration. Nat Rev Neurosci. 2016; 17:251-260. [PubMed: 26988744]

15. Holmes BB, DeVos SL, Kfoury N, Li M, Jacks R, Yanamandra K, Ouidja MO, Brodsky FM, Marasa J, Bagchi DP, Kotzbauer PT, Miller TM, Papy-Garcia D, Diamond MI. Heparan sulfate proteoglycans mediate internalization and propagation of specific proteopathic seeds. Proc Natl Acad Sci U S A. 2013; 110:E3138-E3147. [PubMed: 23898162]

16. Brunden KR, Trojanowski JQ, Lee VMY. Evidence that non-fibrillar tau causes pathology linked to neurodegeneration and behavioral impairments. J Alzheimers Dis. 2008; 14:393-399. [PubMed: 18688089]

17. Patterson KR, Remmers C, Fu Y, Brooker S, Kanaan NM, Vana L, Ward S, Reyes JF, Philibert K, Glucksman MJ, Binder LI. Characterization of prefibrillar Tau oligomers in vitro and in Alzheimer disease. J Biol Chem. 2011; 286:23063-23076. [PubMed: 21550980]

18. Lasagna-Reeves CA, Castillo-Carranza DL, Guerrero-Muoz MJ, Jackson GR, Kayed R. Preparation and characterization of neurotoxic tau oligomers. Biochemistry. 2010; 49:10039_ 10041. [PubMed: 21047142]

19. Flach K, Hilbrich I, Schiffmann A, Gärtner U, Krüger M, Leonhardt M, Waschipky H, Wick L, Arendt T, Holzer M. Tau oligomers impair artificial membrane integrity and cellular viability. J Biol Chem. 2012; 287:43223-43233. [PubMed: 23129775]

20. Lasagna-Reeves CA, Castillo-Carranza DL, Sengupta U, Clos AL, Jackson GR, Kayed R. Tau oligomers impair memory and induce synaptic and mitochondrial dysfunction in wild-type mice. Mol Neurodegener. 2011; 6:39. [PubMed: 21645391] 
21. Violet M, Chauderlier A, Delattre L, Tardivel M, Chouala MS, Sultan A, Marciniak E, Humez S, Binder L, Kayed R, Lefebvre B, Bonnefoy E, Buée L, Galas M-C. Prefibrillar Tau oligomers alter the nucleic acid protective function of Tau in hippocampal neurons in vivo. Neurobiol Dis. 2015; 82:540-551. [PubMed: 26385829]

22. Castillo-Carranza DL, Gerson JE, Sengupta U, Guerrero-Muñoz MJ, Lasagna-Reeves CA, Kayed R. Specific targeting of tau oligomers in Htau mice prevents cognitive impairment and tau toxicity following injection with brain-derived tau oligomeric seeds. J Alzheimers Dis. 2014; 40(Suppl 1):S97-S111. [PubMed: 24603946]

23. Morris M, Maeda S, Vossel K, Mucke L. The many faces of tau. Neuron. 2011; 70:410-426. [PubMed: 21555069]

24. Buée L, Bussière T, Buée-Scherrer V, Delacourte A, Hof PR. Tau protein isoforms, phosphorylation and role in neurodegenerative disorders. Brain Res Brain Res Rev. 2000; 33:95130. [PubMed: 10967355]

25. Lee VM, Goedert M, Trojanowski JQ. Neurodegenerative tauopathies. Annu Rev Neurosci. 2001; 24:1121-1159. [PubMed: 11520930]

26. Ebneth A, Godemann R, Stamer K, Illenberger S, Trinczek B, Mandelkow E. Overexpression of tau protein inhibits kinesin-dependent trafficking of vesicles, mitochondria, and endoplasmic reticulum: Implications for Alzheimer's disease. J Cell Biol. 1998; 143:777-794. [PubMed: 9813097]

27. Trinczek B, Ebneth A, Mandelkow E-M, Mandelkow E. Tau regulates the attachment/detachment but not the speed of motors in microtubule-dependent transport of single vesicles and organelles. $\mathrm{J}$ Cell Sci. 1999; 112(Pt 14):2355-2367. [PubMed: 10381391]

28. Dixit R, Ross JL, Goldman YE, Holzbaur ELF. Differential regulation of dynein and kinesin motor proteins by tau. Science. 2008; 319:1086-1089. [PubMed: 18202255]

29. Vershinin M, Carter BC, Razafsky DS, King SJ, Gross SP. Multiple-motor based transport and its regulation by Tau. Proc Natl Acad Sci U S A. 2007; 104:87-92. [PubMed: 17190808]

30. Dawson HN, Ferreira A, Eyster MV, Ghoshal N, Binder LI, Vitek MP. Inhibition of neuronal maturation in primary hippocampal neurons from tau deficient mice. J Cell Sci. 2001; 114:11791187. [PubMed: 11228161]

31. Nussbaum JM, Schilling S, Cynis H, Silva A, Swanson E, Wangsanut T, Tayler K, Wiltgen B, Hatami A, Rönicke R, Reymann K, Hutter-Paier B, Alexandru A, Jagla W, Graubner S, Glabe CG, Demuth H-U, Bloom GS. Prion-like behaviour and tau-dependent cytotoxicity of pyroglutamylated amyloid- $\beta$. Nature. 2012; 485:651-655. [PubMed: 22660329]

32. Wilcock DM, Lewis MR, Van Nostrand WE, Davis J, Previti ML, Gharkholonarehe N, Vitek MP, Colton CA. Progression of amyloid pathology to Alzheimer's disease pathology in an amyloid precursor protein transgenic mouse model by removal of nitric oxide synthase 2. J Neurosci. 2008; 28:1537-1545. [PubMed: 18272675]

33. Porzig R, Singer D, Hoffmann R. Epitope mapping of mAbs AT8 and Tau5 directed against hyperphosphorylated regions of the human tau protein. Biochem Biophys Res Commun. 2007; 358:644-649. [PubMed: 17499212]

34. Iba M, Guo JL, McBride JD, Zhang B, Trojanowski JQ, Lee VMY. Synthetic tau fibrils mediate transmission of neurofibrillary tangles in a transgenic mouse model of Alzheimer's-like tauopathy. J Neurosci. 2013; 33:1024-1037. [PubMed: 23325240]

35. Dehmelt L, Halpain S. The MAP2/Tau family of microtubule-associated proteins. Genome Biol. 2005; 6:204. [PubMed: 15642108]

36. Lee MK, Tuttle JB, Rebhun LI, Cleveland DW, Frankfurter A. The expression and posttranslational modification of a neuron-specific beta-tubulin isotype during chick embryogenesis. Cell Motil Cytoskeleton. 1990; 17:118-132. [PubMed: 2257630]

37. Greenberg SG, Davies P, Schein JD, Binder LI. Hydrofluoric acid-treated tau PHF proteins display the same biochemical properties as normal tau. J Biol Chem. 1992; 267:564-569. [PubMed: 1370450]

38. Holmes BB, Furman JL, Mahan TE, Yamasaki TR, Mirbaha H, Eades WC, Belaygorod L, Cairns NJ, Holtzman DM, Diamond MI. Proteopathic tau seeding predicts tauopathy in vivo. Proc Natl Acad Sci U S A. 2014; 111:E4376-E4385. [PubMed: 25261551] 
39. Wu JW, Herman M, Liu L, Simoes S, Acker CM, Figueroa H, Steinberg JI, Margittai M, Kayed R, Zurzolo C, Di Paolo G, Duff KE. Small misfolded Tau species are internalized via bulk endocytosis and anterogradely and retrogradely transported in neurons. J Biol Chem. 2013; 288:1856-1870. [PubMed: 23188818]

40. Usenovic M, Niroomand S, Drolet RE, Yao L, Gaspar RC, Hatcher NG, Schachter J, Renger JJ, Parmentier-Batteur S. Internalized tau oligomers cause neurodegeneration by inducing accumulation of pathogenic tau in human neurons derived from induced pluripotent stem cells. $\mathrm{J}$ Neurosci. 2015; 35:14234-14250. [PubMed: 26490863]

41. Nussbaum JM, Seward ME, Bloom GS. Alzheimer disease: A tale of two prions. Prion. 2013; 7:14-19. [PubMed: 22965142]

42. Alonso AD, Grundke-Iqbal I, Barra HS, Iqbal K. Abnormal phosphorylation of tau and the mechanism of Alzheimer neurofibrillary degeneration: Sequestration of microtubule-associated proteins 1 and 2 and the disassembly of microtubules by the abnormal tau. Proc Natl Acad Sci U S A. 1997; 94:298-303. [PubMed: 8990203]

43. Seward ME, Swanson E, Norambuena A, Reimann A, Cochran JN, Li R, Roberson ED, Bloom GS. Amyloid- $\beta$ signals through tau to drive ectopic neuronal cell cycle re-entry in Alzheimer's disease. J Cell Sci. 2013; 126:1278-1286. [PubMed: 23345405]

44. Norambuena A, Wallrabe H, McMahon L, Silva A, Swanson E, Khan SS, Baerthlein D, Kodis E, Oddo S, Mandell JW, Bloom GS. mTOR and neuronal cell cycle reentry: How impaired brain insulin signaling promotes Alzheimer's disease. Alzheimers Dement. 2017; 13:152-167. [PubMed: 27693185]

45. Augustinack JC, Schneider A, Mandelkow E-M, Hyman BT. Specific tau phosphorylation sites correlate with severity of neuronal cytopathology in Alzheimer's disease. Acta Neuropathol. 2001; 103:26-35.

46. Weaver CL, Espinoza M, Kress Y, Davies P. Conformational change as one of the earliest alterations of tau in Alzheimer's disease. Neurobiol Aging. 2000; 21:719-727. [PubMed: 11016541]

47. Wolozin BL, Pruchnicki A, Dickson DW, Davies P. A neuronal antigen in the brains of Alzheimer patients. Science. 1986; 232:648-650. [PubMed: 3083509]

48. Hyman BT, Van Hoesen GW, Wolozin BL, Davies P, Kromer LJ, Damasio AR. Alz-50 antibody recognizes Alzheimer-related neuronal changes. Ann Neurol. 1988; 23:371-379. [PubMed: 3382173]

49. Petry FR, Pelletier J, Bretteville A, Morin F, Calon F, Hébert SS, Whittington RA, Planel E. Specificity of anti-tau antibodies when analyzing mice models of Alzheimer's disease: Problems and solutions. PLoS One. 2014; 9:e94251. [PubMed: 24788298]

50. Otvos L, Feiner L, Lang E, Szendrei GI, Goedert M, Lee VM. Monoclonal antibody PHF-1 recognizes tau protein phosphorylated at serine residues 396 and 404. J Neurosci Res. 1994; 39:669-673. [PubMed: 7534834]

51. Zempel H, Mandelkow E. Lost after translation: Missorting of Tau protein and consequences for Alzheimer disease. Trends Neurosci. 2014; 37:721-732. [PubMed: 25223701]

52. Ittner LM, Ke YD, Delerue F, Bi M, Gladbach A, van Eersel J, Wölfing H, Chieng BC, Christie MJ, Napier IA, Eckert A, Staufenbiel M, Hardeman E, Götz J. Dendritic function of tau mediates amyloid-beta toxicity in Alzheimer's disease mouse models. Cell. 2010; 142:387-397. [PubMed: 20655099]

53. Sohn PD, Tracy TE, Son H-I, Zhou Y, Leite REP, Miller BL, Seeley WW, Grinberg LT, Gan L. Acetylated tau destabilizes the cytoskeleton in the axon initial segment and is mislocalized to the somatodendritic compartment. Mol Neurodegener. 2016; 11:47. [PubMed: 27356871]

54. Zempel H, Thies E, Mandelkow E, Mandelkow E-M. Abeta oligomers cause localized Ca(2+) elevation, missorting of endogenous Tau into dendrites, Tau phosphorylation, and destruction of microtubules and spines. J Neurosci. 2010; 30:11938-11950. [PubMed: 20826658]

55. Baas PW, Deitch JS, Black MM, Banker GA. Polarity orientation of microtubules in hippocampal neurons: Uniformity in the axon and nonuniformity in the dendrite. Proc Natl Acad Sci U S A. 1988; 85:8335-8339. [PubMed: 3054884] 
56. Heidemann SR, Landers JM, Hamborg MA. Polarity orientation of axonal microtubules. J Cell Biol. 1981; 91:661-665. [PubMed: 6173385]

57. Maday S, Twelvetrees AE, Moughamian AJ, Holzbaur ELF. Axonal transport: Cargo-specific mechanisms of motility and regulation. Neuron. 2014; 84:292-309. [PubMed: 25374356]

58. Das U, Wang L, Ganguly A, Saikia JM, Wagner SL, Koo EH, Roy S. Visualizing APP and BACE-1 approximation in neurons yields insight into the amyloidogenic pathway. Nat Neurosci. 2016; 19:55-64. [PubMed: 26642089]

59. Trinczek B, Ebneth A, Mandelkow E-M, Mandelkow E. Tau regulates the attachment/detachment but not the speed of motors in microtubule-dependent transport of single vesicles and organelles. J Cell Sci. 1999; 112(Pt 14):2355-2367. [PubMed: 10381391] 

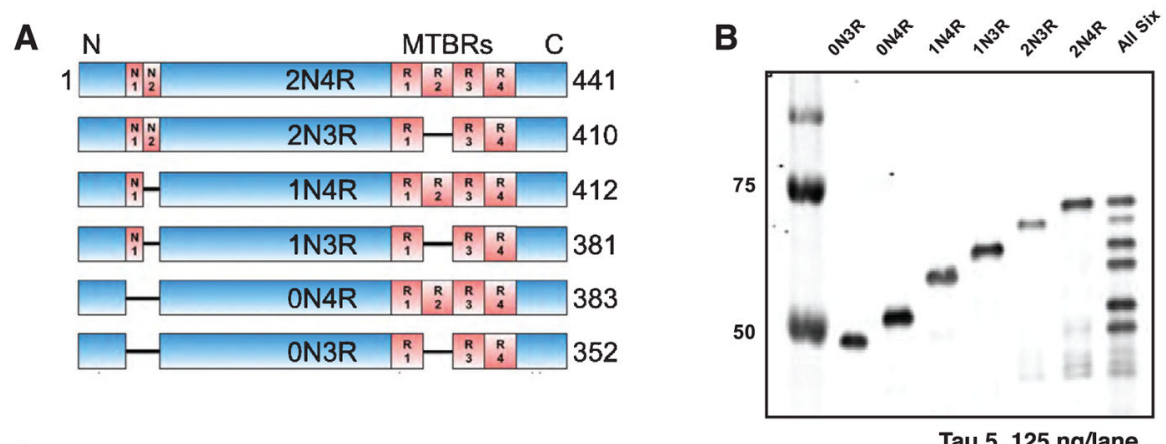

C

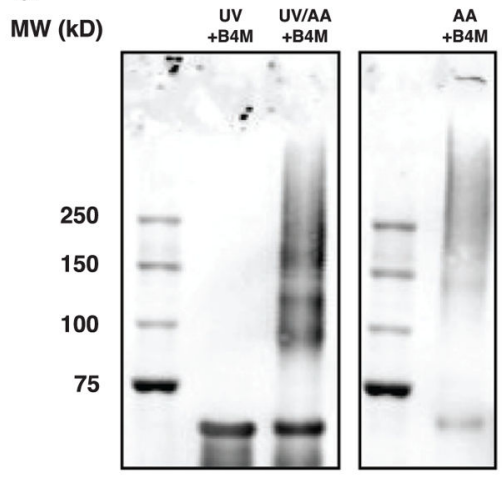

D

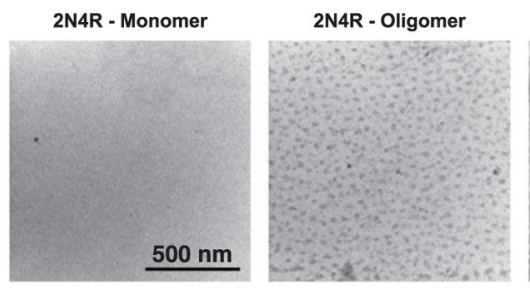

2N4R - Fibrils

Fig. 1.

Characterization of Tau oligomers A) Tau is found in the central nervous system as six alternatively-spliced isoforms, characterized by the inclusion of 0,1 , or $2 \mathrm{~N}$-terminal inserts, and 3 or 4 C-terminal microtubule-binding repeats (MTBRs). B) Western blotting of Nterminal his-tagged recombinant tau isoforms probed with the Tau 5 antibody. C) Tau 5 western blot of monomeric, oligomeric, and fibrillar 2N4R tau. D) Negative stain transmission electron micrographs of 2N4R monomeric, oligomeric, and fibrillar tau. 


\section{A Extracellular 2N4R tau oligomers}

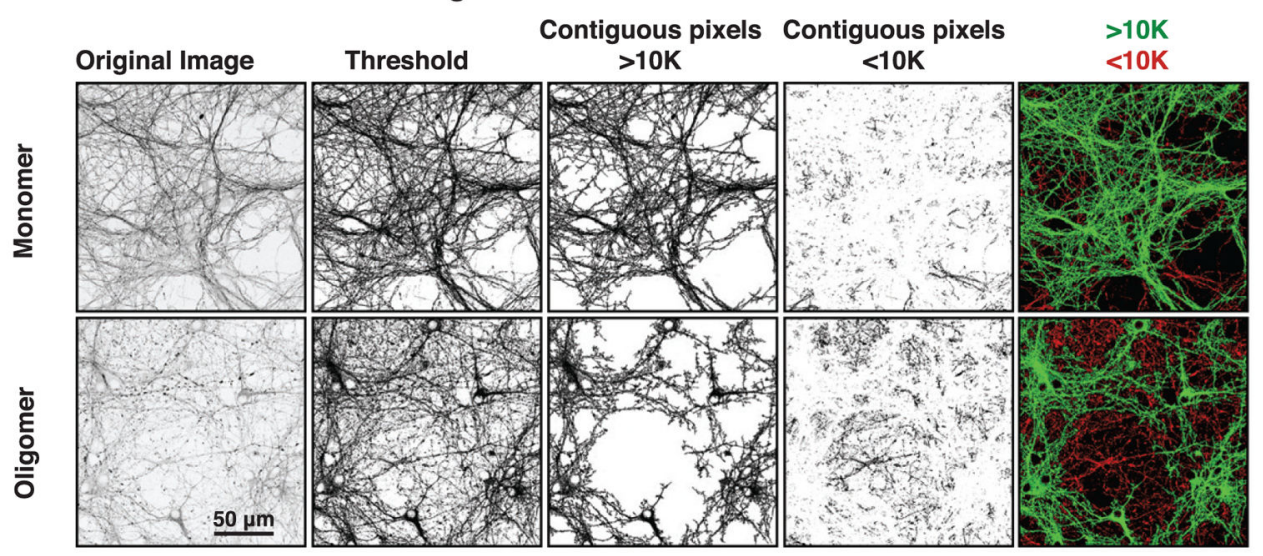

B Extracellular 2N4R oligomers

C
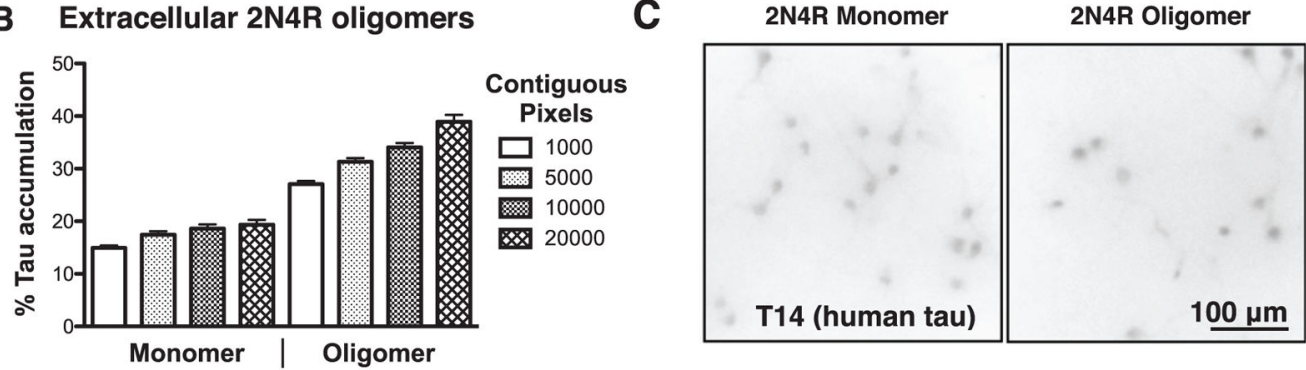

Fig. 2.

Image analysis of tau-treated neurons. A) Representative immunofluorescence images of primary neurons stained for total tau with Tau 5 after treatment with monomeric or oligomeric extracellular tau. Fluorescence micrographs were thresholded to indicate pixels above background intensity, segregated into groups of $>10,000$ or $<10,000$ contiguous pixels above the threshold intensity, then pseudo-colored green to indicate intact staining $(>10,000$ contiguous pixels) or red to indicate disrupted staining ( $<10,000$ contiguous pixels). B) Test of various pixel size cutoffs in monomer or oligomer treated neurons. 10,000 contiguous pixels was chosen as the cutoff between intact and disrupted tau for all subsequent assays. C) Monomer and oligomer treated cells probed with antibodies for human tau (T14) exhibit only background staining. 


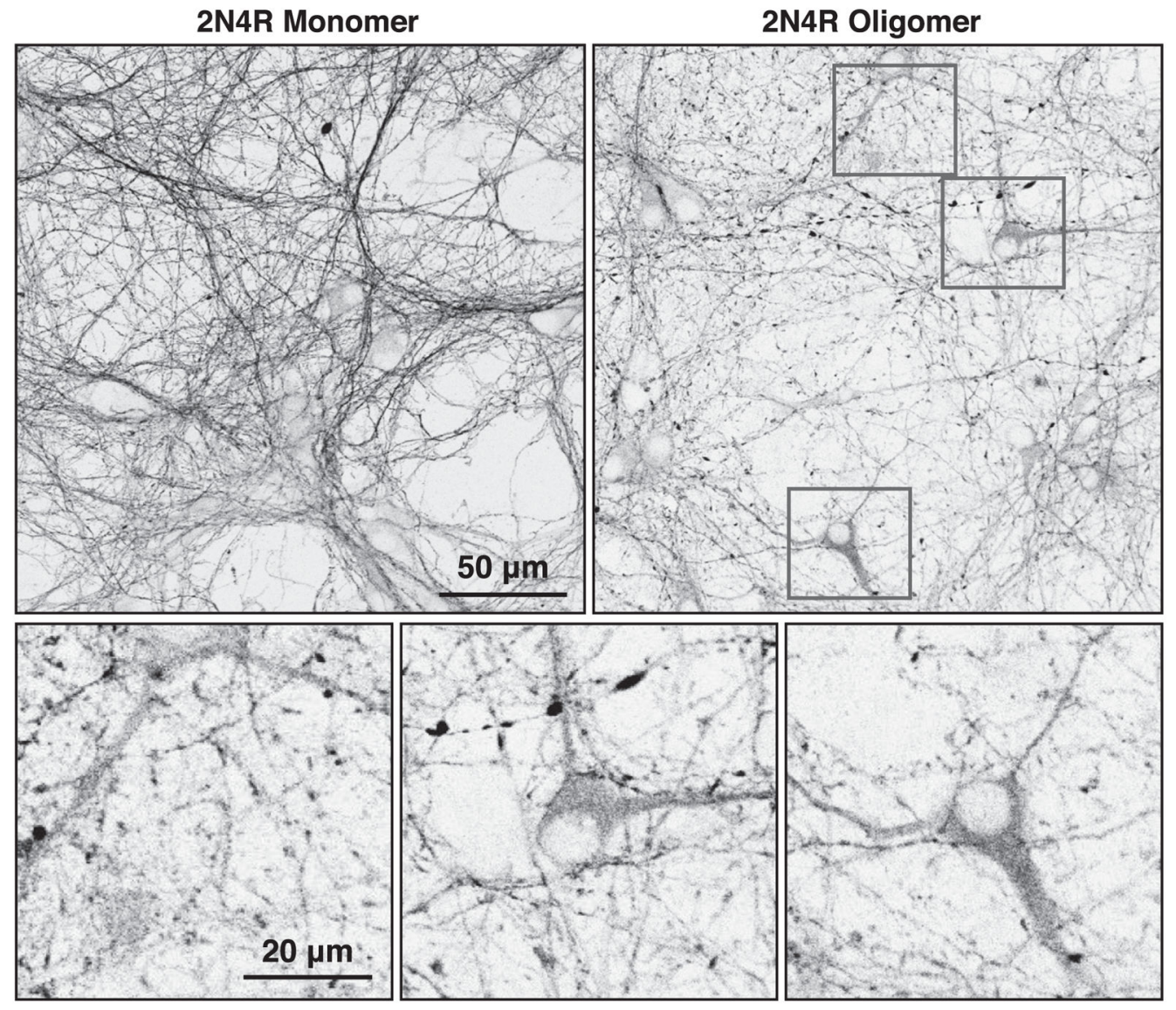

Fig. 3.

Extracellular tau oligomers induce endogenous tau to aggregate in axons and invade the somatodendritic compartment. High-resolution immunofluorescence micrographs of primary mouse cortical neurons are shown here. Relatively continuous axonal tau staining and minimal somatodendritic tau staining was observed following exposure to 2N4R monomers, while 2N4R oligomer-treated cells developed tau puncta and varicosities in axons, and striking tau accumulation in the somatodendritic compartment. 
A

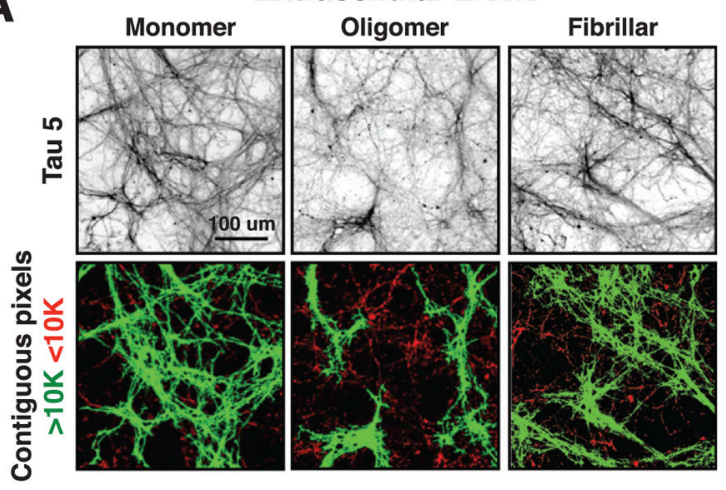

C

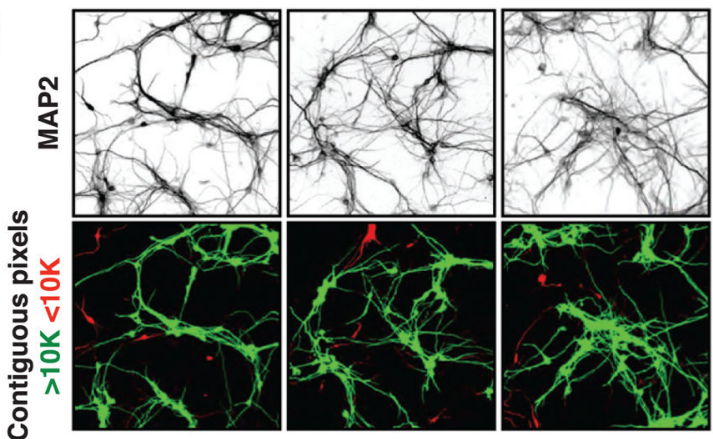

E

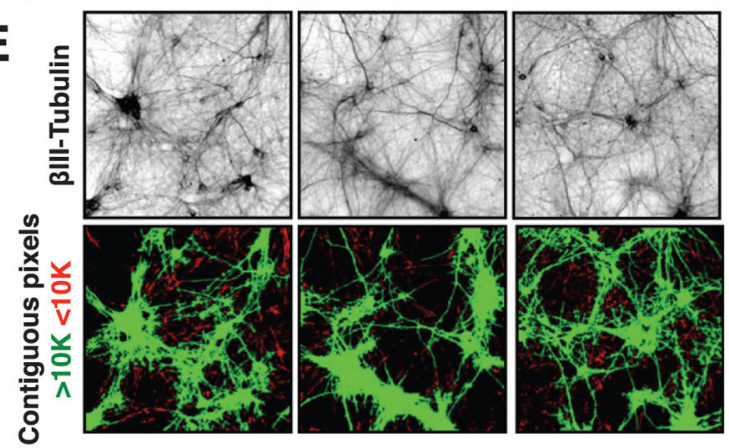

B
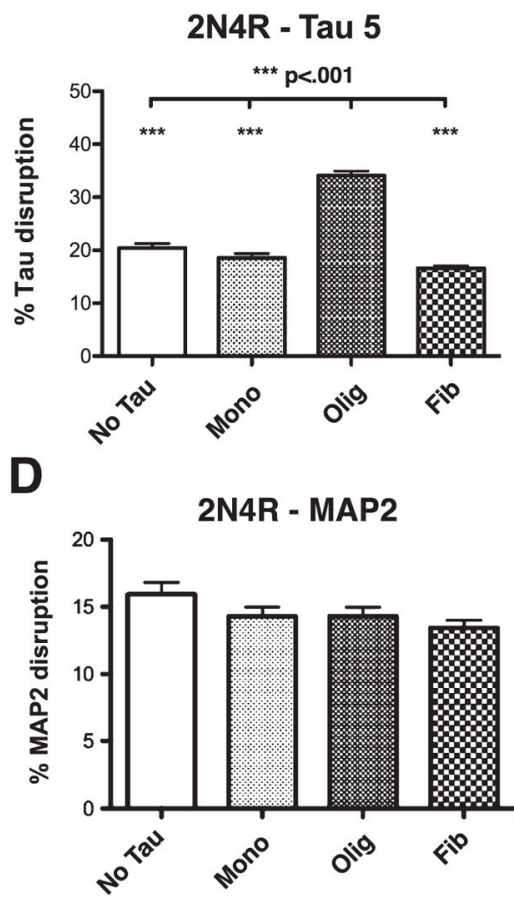

$\mathbf{F}$

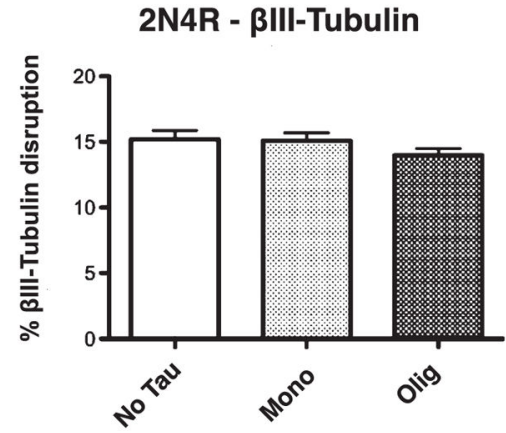

Fig. 4.

Extracellular 2N4R tau oligomers disrupt endogenous wild-type tau. A, B) Extracelluar 2N4R oligomers, but not monomers or fibrils, disrupt endogenous tau, as visualized by Tau 5 staining. C-F) No disruption of MAP2 or BIII tubulin was observed after treatment of neurons with monomeric, oligomeric or fibrillar 2N4R tau. Error bars represent SEM. 

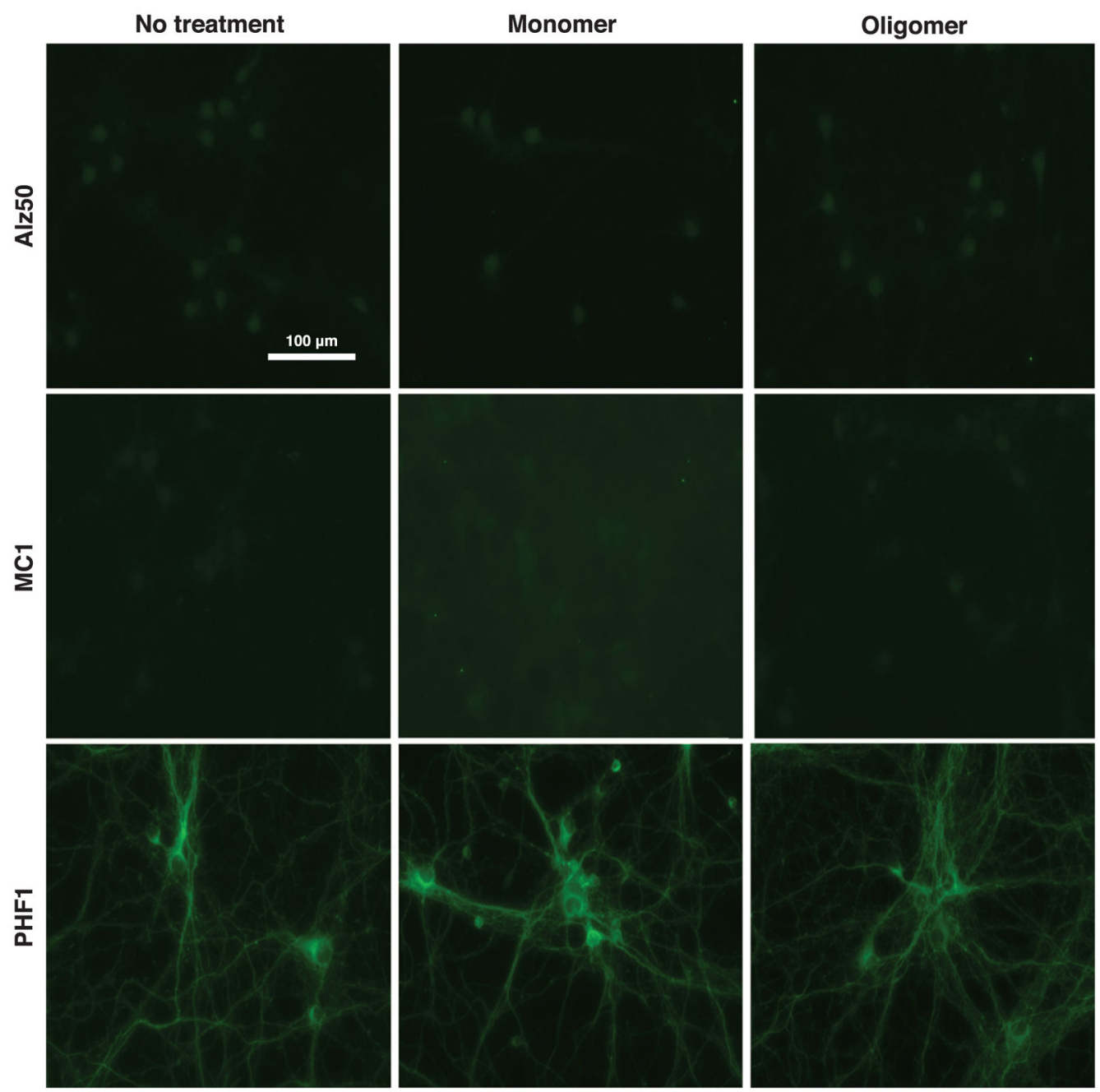

Fig. 5.

Extracellular tau oligomers do no rapidly induce selective AD-like conformational changes or phosphorylation of endogenous intracellular tau. No immunoreactivity was observed for the conformation-specific antibodies, Alz50 or MC1, in untreated neurons or those exposed to extracellular 2N4R tau monomers or oligomers. Additionally, a low constant level of PHF-1 was observed for untreated, monomer-treated and oligomer-treated cells. 
A Extracellular 2N3R
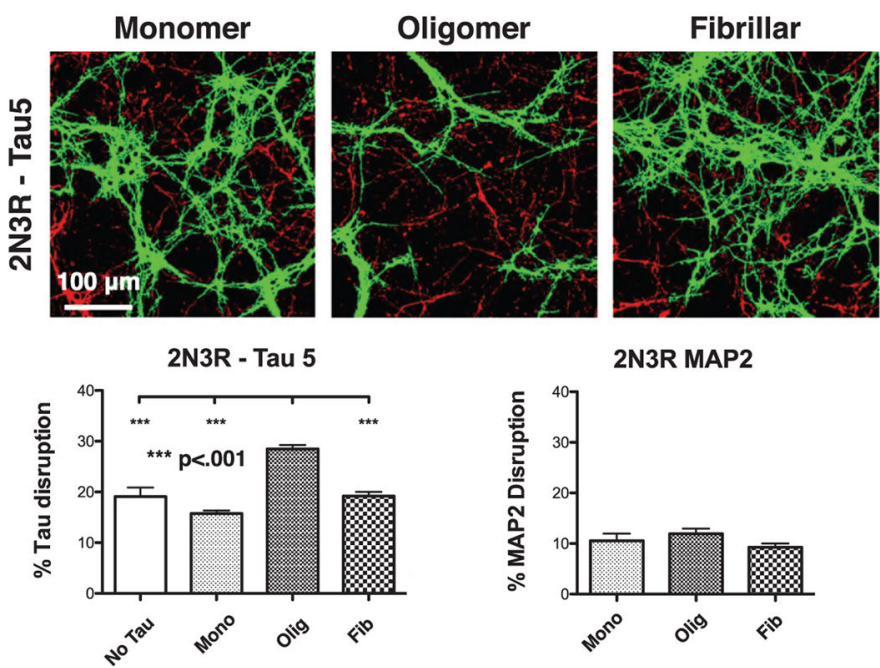

2N3R MAP2

B Extracellular $1 \mathrm{~N}$
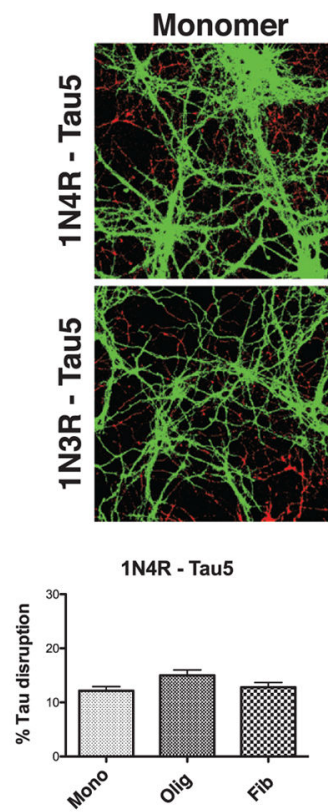

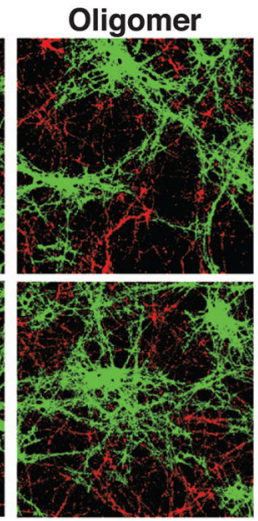

1N3R - Tau 5

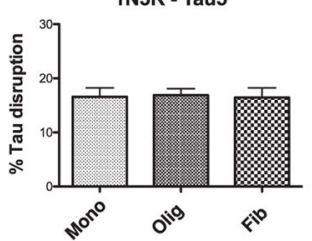

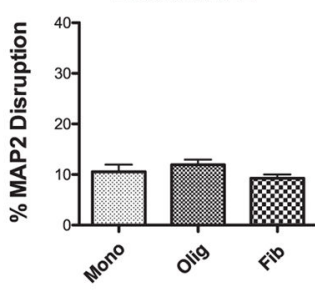

C Extracellular on
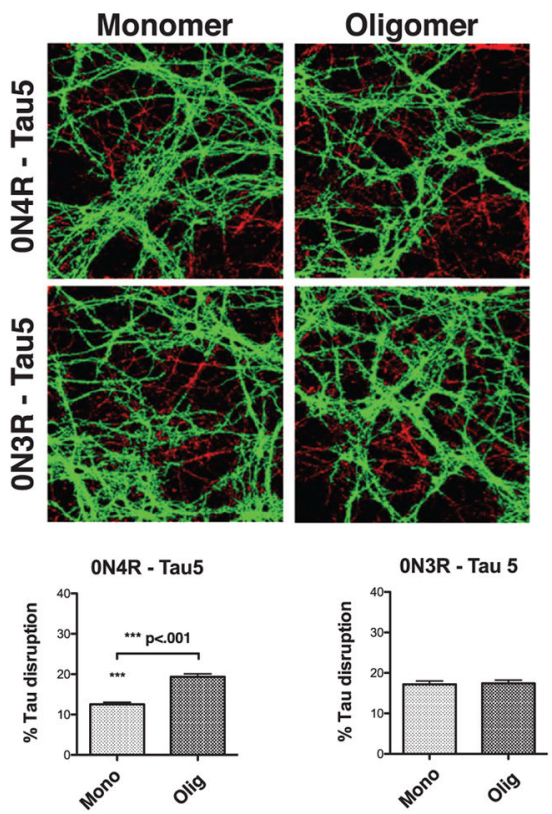

Fig. 6.

The extent of intracellular tau disruption in tau oligomer treated-cells varies by the tau isoform composition of the oligomers. A) Neurons treated with oligomeric, but not monomeric or fibrillar 2N3R tau, show aggregation of tau, but not of MAP2. B) Neurons treated with extracellular monomers, oligomers, of fibrils composed of $1 \mathrm{~N}$ tau isoforms exhibit no significant disruption of endogenous tau. C) Oligomers composed of 0N4R, but not 0N3R tau caused aggregation of endogenous axonal tau. Error bars represent SEM. 
A Extracellular 2N3R/4R treatment
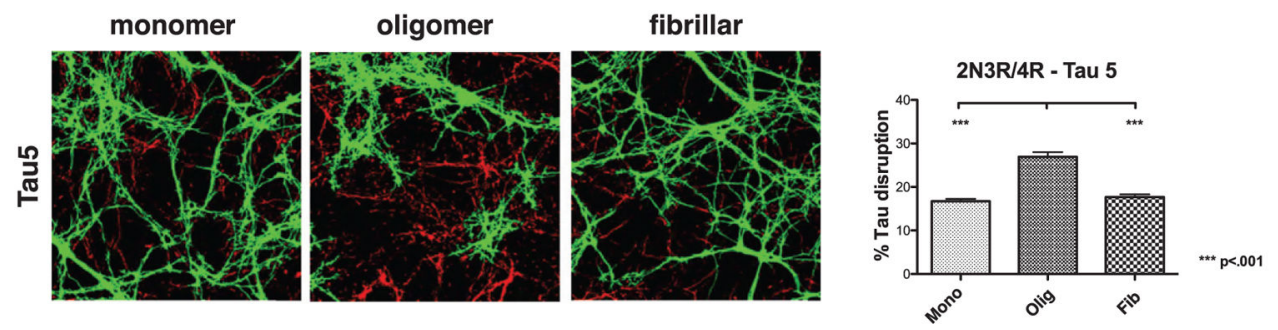

B All Six Isoforms treatment
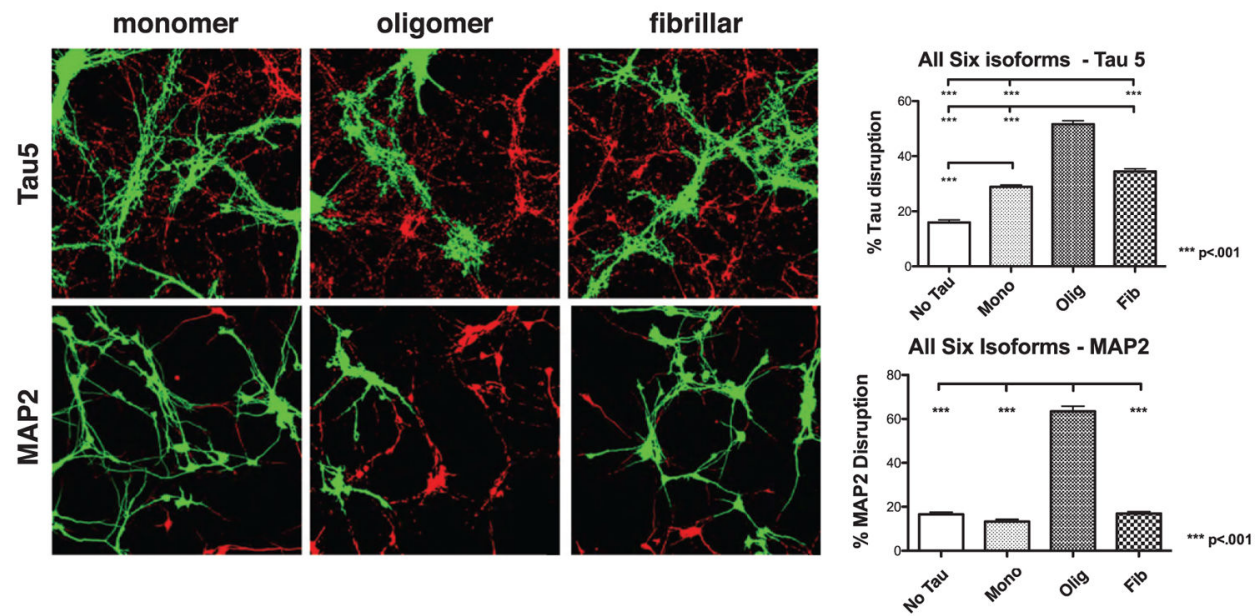

Fig. 7.

Extracellular oligomers of mixed isoform composition cause endogenous tau aggregation. A) Neurons treated with oligomers, but not monomers or fibrils, of a 1:1 mixture of 2N3R and 2N4R tau caused aggregation of endogenous axonal tau. B) Neurons exposed to an equimolar mix of all 6 tau isoforms developed endogenous tau aggregates, especially when the extracellular tau was oligomeric. Treatment with oligomers of all 6 isoforms additionally caused extensive aggregation of MAP2. Error bars represent SEM. 

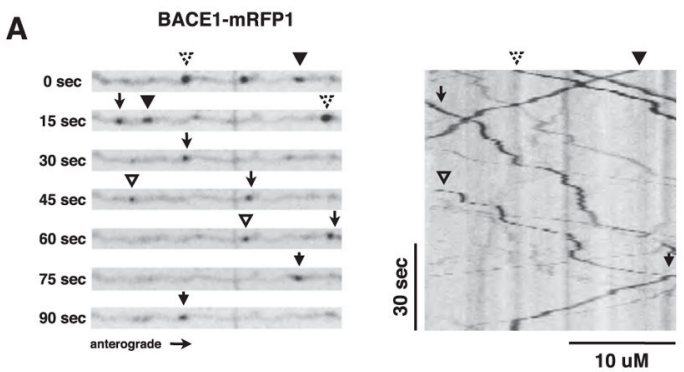

B
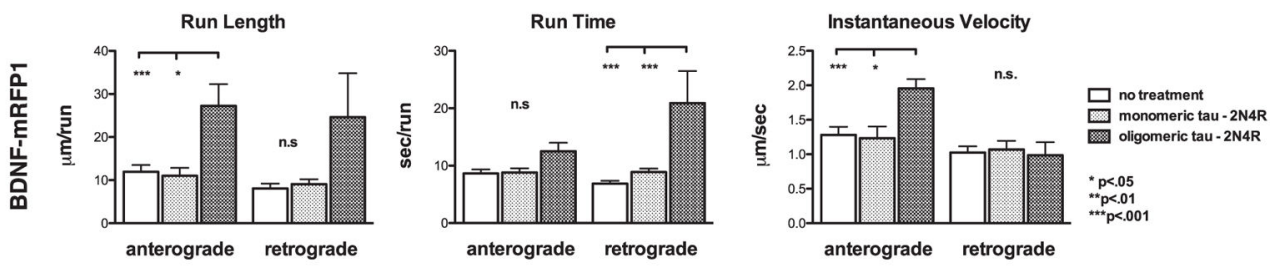

C
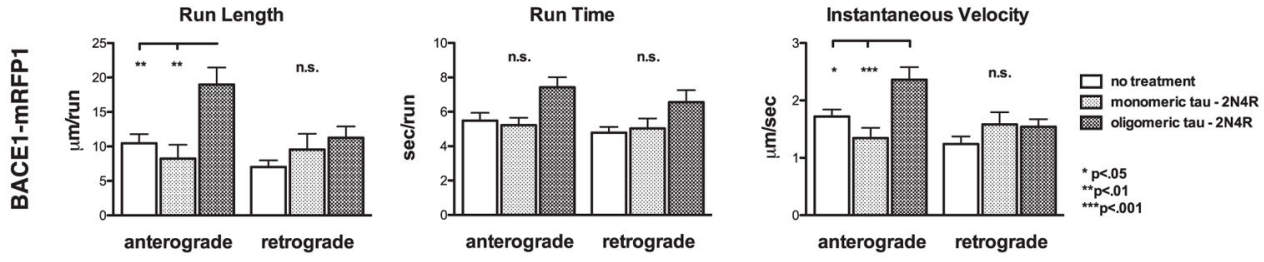

D
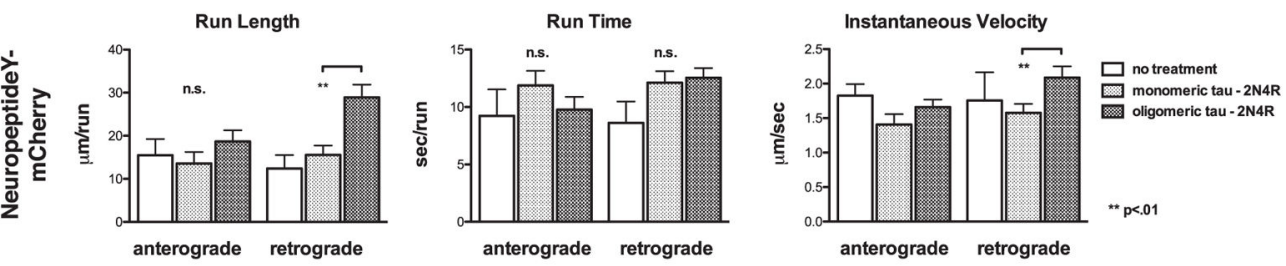

Fig. 8.

Tau oligomer exposure induces disruption of fast axonal transport. A) Transport parameters were analyzed by live cell, time lapse fluorescence microscopy (left) followed by kymograph analysis (right). Run length, run time, and instantaneous velocity were quantified for neurons transfected with expression vectors for B) BDNF-mRFP1, C) BACE1-mRFP1. and D) NeuropeptideY-mCherry. Error bars represent SEM. 
A
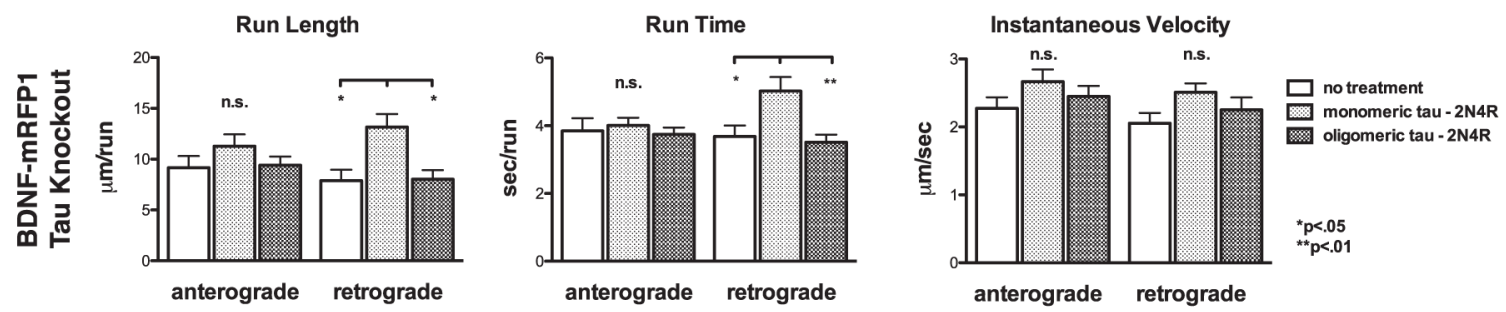

B
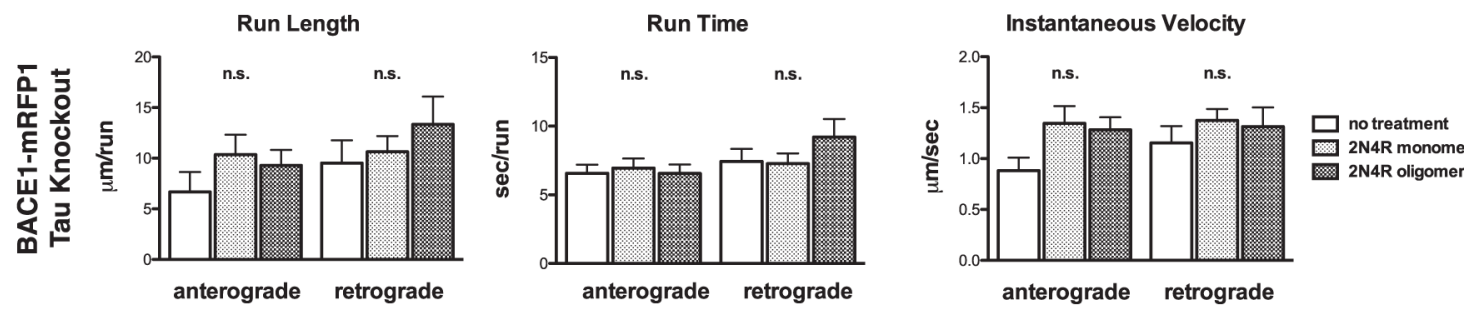
2N4R monomer tx

C
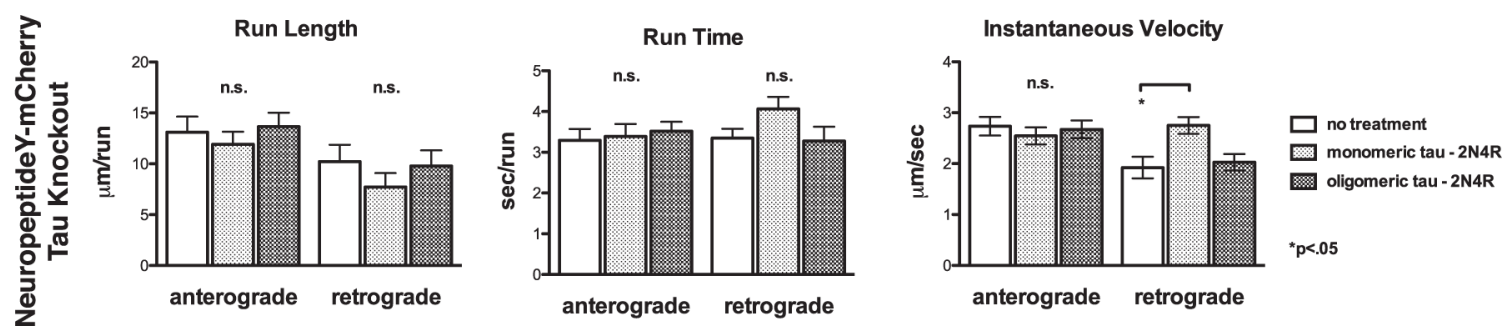

Fig. 9.

Robust disruption of fast axonal transport disruption by extracellular tau oligomers requires endogenous tau. Transport parameters were analyzed in tau knockout neurons by live cell, time lapse fluorescence microscopy followed by kymograph analysis, as for Fig. 8. Run length, run time, and instantaneous velocity were quantified for neurons transfected with expression vectors for A) BDNF-mRFP1, B) BACE1-mRFP1, and C) NeuropeptideYmCherry. Error bars represent SEM. 